\title{
Particle acceleration in stochastic current sheets in stressed coronal active regions
}

\author{
R. Turkmani ${ }^{1}$, P. J. Cargill ${ }^{1}$, K. Galsgaard ${ }^{2}$, L. Vlahos ${ }^{3}$, and H. Isliker ${ }^{3}$ \\ 1 Space and Atmospheric Physics group, Imperial College, London, UK \\ e-mail: [r.turkmani;p.cargill]@ic.ac.uk \\ 2 Niels Bohr Institute, Copenhagen, Denmark \\ e-mail: kg@astro.ku.dk \\ 3 Department of Physics, University of Thessaloniki, 54124 Thessaloniki, Greece \\ e-mail: [vlahos;isliker] @astro.auth.gr
}

Received 1 June 2005 / Accepted 6 December 2005

\begin{abstract}
Aims. To perform numerical experiments of particle acceleration in the complex magnetic and electric field environment of the stressed solar corona.

Methods. The magnetic and electric fields are obtained from a 3-D MHD experiment that resembles a coronal loop with photospheric regions at both footpoints. Photospheric footpoint motion leads to the formation of a hierarchy of stochastic current sheets. Particles (protons and electrons) are traced within these current sheets starting from a thermal distribution using a relativistic test particle code.

Results. In the corona the particles are subject to acceleration as well as deceleration, and a considerable portion of them leave the domain having received a net energy gain. Particles are accelerated to high energies in a very short time (both species can reach energies up to $100 \mathrm{GeV}$ within $5 \times 10^{-2} \mathrm{~s}$ for electrons and $5 \times 10^{-1} \mathrm{~s}$ for protons). The final energy distribution shows that while one quarter of the particles retain their thermal distribution, the rest have been accelerated, forming a two-part power law. Accelerated particles are either trapped within electric field regions of opposite polarities, or escape the domain mainly through the footpoints. The particle dynamics are followed in detail and it is shown how this dynamic affects the time evolution of the system and the energy distribution. The scaling of these results with time and length scale is examined and the Bremstrahlung signature of X-ray photons resulting from escaping particles hitting the chromosphere is calculated and found to have a main power law part with an index $\gamma=-1.8$, steeper than observed. Possible resolutions of this discrepency are discussed.
\end{abstract}

Key words. Sun: flares - Sun: particle emission - Sun: X-rays, gamma rays

\section{Introduction}

In the past 15 years high temporal, spatial and spectral resolution observations of radiation from energetic particles in solar flares, especially from the Yohkoh and Ramaty High Energy Solar Spectroscopic Imager (RHESSI) missions, have greatly enhanced our knowledge of the principal characteristics of the particle acceleration process(es). The following results are now accepted: (a) a large number of electrons (up to $10^{37}$ pers above $20 \mathrm{keV}$ ) are accelerated in a very short time (sub-second) to energies in excess of a few $\mathrm{MeV}$; (b) a significant number of ions (up to $10^{35}$ pers above $1 \mathrm{Mev}$ ) are accelerated to in excess of $1 \mathrm{MeV}$; (c) a fraction of these electrons and ions are accelerated to relativistic energies; (d) the typical spectra consist of a thermal part at low energies, and a one or two part powerlaw at higher energy; (e) there is acceleration going on both before the main impulsive phase and well into the decay phase; (f) there is a delay in the acceleration time between electrons and ions, with electrons being accelerated faster than ions (e.g. Lin et al. 2003). Other constraints also exist, such as the temporal evolution of spectral indexes (e.g. Grigis \& Benz 2004) and the presence in some cases of a separation between the X-ray and $\gamma$-ray emission sites (e.g. Hurford et al. 2003).

In general terms, these results have affirmed the longstanding semi-conjecture that energetic particles may account for the largest share of the energy budget of a flare. Indeed in some cases up to $50 \%$ of the energy released by solar flares seems to go to electrons alone (e.g. Miller et al. 1997). When one adds energetic ions, especially the hard-to-determine component with energies under a few $\mathrm{MeV}$, the energy requirements are significant.

These results pose a formidable challenge for theoretical models, not least the requirement to convert so much electromagnetic energy into energetic particle kinetic energy. Another 
challenge is that, despite the high spatial and temporal resolution of current observations, the majority of flares (medium and small ones) are still probably not yet adequately resolved. (We note though that big flares appear to be relatively well resolved, and structures like loops, footpoints and looptops are apparent. As a result they have tended to be the focus of theoretical modelling.) It is plausible that the energy release processes occurs on sub-scales well beyond our observational means and theoretical models have shown that small scale energy releases can answer many questions, in particular the fast timescales (e.g. Miller et al. 1997; Arzner \& Vlahos 2004; Turkmani et al. 2005; Dmitruk et al. 2003).

However, models for flare particle acceleration are confronted by an imposing difficulty, namely the disparity between two scales: the global magnetic structure of a flare is determined by the evolution of the meso- and macro-scale corona, and is describable using a magnetohydrodynamic (MHD) approach, whereas particle acceleration is a kinetic process, occurring on very small scales, such as in and around current sheets. One approach addresses particle acceleration in idealised field geometries and plasma environments. Examples include acceleration at a current sheet by direct electric field or turbulence, and stochastic acceleration of electrons and ions inside a loop filled with low-frequency waves (Miller et al. 1997). Turbulent acceleration in particular has had some successes, but suffers from the difficulty that the source and the evolution of the required MHD waves is not clearly defined, or related to the current sheet(s) believed to be responsible for the energy released during a flare.

A first step toward combining the global coronal magnetic field with acceleration physics used Cellular Automata (CA) models (Anastasiadis \& Vlahos 1994; Anastasiadis et al. 1997; Vlahos et al. 2004; Anastasiadis et al. 2004). In these models an active region was driven by turbulent photospheric motions, and gradually developed unstable current sheets at random points. These then accelerate the particles. Of course the CA approach does not contain the detailed MHD description of the entire coronal configuration.

In a recent letter (Turkmani et al. 2005) we took an important step forward. A fully three-dimensional time-dependent MHD model was used to describe the large scale coronal structure as it responds to photospheric driving, and then followed the evolution of particles within the resulting magnetic and electric fields. We showed that this presents an efficient particle acceleration mechanism that has the potential to accelerate both ions and electrons to relativistic energies in times of order $10^{-2} \mathrm{~s}$ for electrons and $10^{-1} \mathrm{~s}$ for protons forming power law distributions in energy. In this paper, we aim to (a) document fully the methodology and basic results that could not be included in the earlier paper for reasons of space; (b) extend the results to look at a broader range of parameters to demonstrate the robustness of the acceleration process; and (c) calculate radiative signatures of the accelerated electrons.

The models are described in Sect. 2. The results are described in Sect. 3 and the Discussion and conclusions are in Sect. 4.

\section{Model description}

The MHD coronal model used in this paper is based on that presented in Galsgaard \& Nordlund (1996) and Galsgaard (2002), but in the interests of clarity, a general description is provided. The three-dimensional time-dependent MHD equations are solved in a cartesian box with model photospheres at either end. An energy equation with anisotropic heat condition and optical thin radiation is included. Between photosphere and corona there is a stratified atmosphere (the gravitational force is modelled by a sine function, vanishing at the centre of the computational box), so that the density profile is a hyperbolic tangent. At the start of each simulation, there is a uniform magnetic field extending between the two photospheric regions. In order to relate the simulations to observed coronal loop structures, the simulation box is 20 times longer than it is wide. The coronal density is $10^{3}$ times smaller than that in the photosphere, implying an Alfvén speed in the corona approximately 30 times larger than in the photosphere. (This small ratio is due to computational considerations that require the Alfvén velocity be small enough to permit a practical timestep.)

This initial state is perturbed by imposing simple sinusoidal shear motions on the magnetic field at the two boundaries. Their wavelength is equal to the transverse length, while their phase, orientation and direction are random. This, in a simple manner, represents the advection of magnetic flux due to convective motions, and injects energy into the corona. The coronal field responds to these boundary motions, with the Lorentz force determining its evolution. After some time coronal current sheet formation occurs. An exponential growth of current density implies that after a finite time (which scales logarithmically with the magnetic resistivity), diffusion must become important. As reconnection commences, plasma jets are formed and eventually their momentum is sufficient to strongly perturb the neighbouring plasma, creating secondary current concentrations. A turbulent cascade is thus initiated so that throughout the simulation, energy is injected on large scales, but cascades through a turbulent process to the shortest possible length scale where it is dissipated in numerous small current concentrations randomly distributed throughout the volume. The consequence of this is a magnetic dissipation that is independent of the value of the resistivity (Hendrix \& Van Hoven 1996).

The simplest option in such a simulation is to include a constant resistivity, but numerically this is not sensible since it implies that even well-resolved current structures diffuse away. Resolving the inertial range of MHD turbulence will then only be possible for very low resistivites and a correspondingly high numerical resolution. For three-dimensional simulations this is far too expensive in terms of computer time. An alternative approach used here is to apply magnetic diffusion in a way that prevents numerical problems such as infinite steepening from occurring. This is particularly important in regions where the fast mode and advection speeds change, and also where the magnetic field is compressed in the direction perpendicular to the magnetic field vector. This way the numerical resistivity can be localised to the regions where numerical problems may arise. Further localisation is achieved through the use of special high-order operators. This implies that structures are allowed 
to evolve nearly ideally down to length scales close to the numerical resolution before diffusion becomes important for the dynamical evolution. The numerical resistivity has a very low value outside the current sheets regions, which is set to zero there, before being used for the test-particles experiment to avoid exaggerated acceleration.

For the purposes of this paper, we use the output of the MHD model, specifically the three-dimensional electric and magnetic fields, as a basis for studying particle acceleration. We do not track particles in evolving fields; rather look at "snapshots". This is justified by the separation of timescales for acceleration $(<1 \mathrm{~s})$ from the characteristic coronal evolution time $(>1 \mathrm{~s})$. We consider acceleration only in the coronal part of the model, so ignore any current sheets in the photsophere.

The MHD simulation was performed on a numerical grid with 200 points between the photospheric boundaries (the $x$-coordinate), and 60 points in each of the transverse directions (the $y$ - and $z$-coordinates). Only the coronal portion is considered, so that in terms of a dimensionless length, our electric and magnetic fields are confined to $\boldsymbol{L}=\left(L_{x}, L_{y}, L_{z}\right)=$ $(1.6,0.1,0.1)$. Physical lengths are obtained by multiplying $\boldsymbol{L}$ by a factor $L$. Most of our results use $L=10^{9} \mathrm{~cm}$, but in Sect. 3.3 we consider other values. The value of the background coronal density is taken to be $10^{10} \mathrm{~cm}^{-3}$, the initial background magnetic field is taken to be $B=100 \mathrm{G}$ and the coronal plasma beta is equal to 0.04 .

In our earlier paper (Turkmani et al. 2005), we examined the electric field (both resistive and inductive) arising in this model, and their potential as particle accelerators. The inductive field appeared to be negligible (especially for high energy particles) because its component parallel to the magnetic field is zero. In this paper, we use only the resistive field but refer to the impact of including the inductive field when relevant.

This resistive electric field is distributed over the domain in the form of a hierarchy of current sheets. Figure 1 shows the snapshot of these current sheets from the MHD model that is used in this paper: details are given in the caption. In between the current sheets no electric field exist, while the electric field inside the current sheets takes on values between $\pm 3 \times 10^{-2}$ statvolt $/ \mathrm{cm}$. The average absolute value of the electric field is $5 \times 10^{-4}$ statvolt $/ \mathrm{cm}$.

This can be seen more clearly in Fig. 2a where a 1D sample of the $x$-component of the electric field along the domain is shown. The distribution function of the values of the magnitude of the electric field is shown in Fig. 2b. The distribution has a power law component with an index value of -2.8 which terminates at a cut-off at the highest values.

To model particle acceleration, test particles (both electrons and protons are considered) are run through the fields produced by the MHD code by solving numerically the relativistic equations of motion using a 4th order Runge-Kutta method with an adaptive step-size. The particles are injected in the MHD domain simultaneously and are considered as "lost" when they leave the simulation box and are not replaced. The test particle approximation implies that the energy gained by these particles does not feed back into the MHD calculations, and the MHD electromagnetic fields are unmodified by any currents

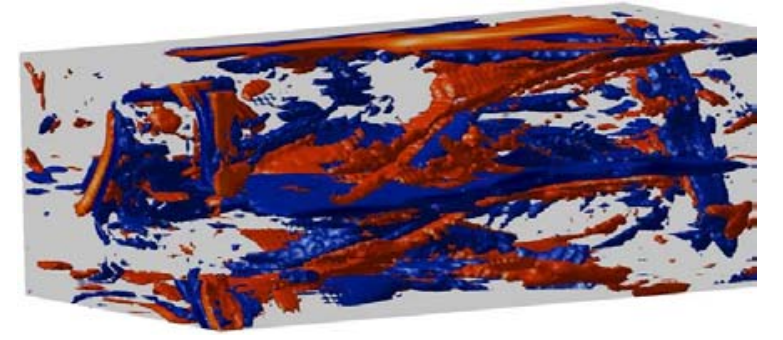

Fig. 1. Snapshots of the resistive electric field configurations within the coronal volume, as calculated from the global MHD model. The blue and red regions represent electric field regions that point towards the left and right foot points respectively. The details of the model are described in the text.
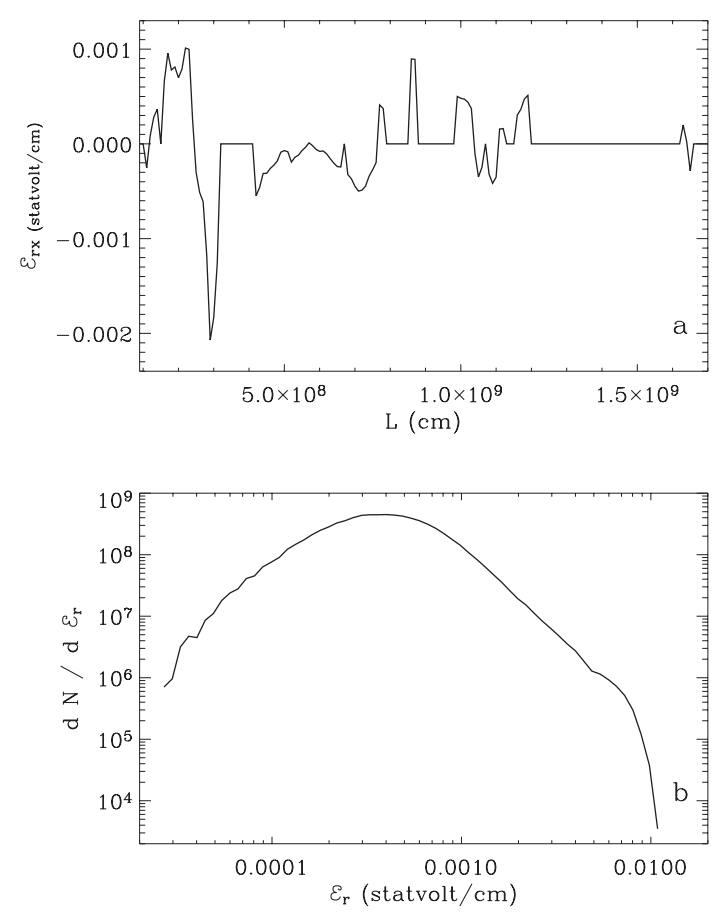

Fig. 2. a) An arbitrary 1-D sample of the resistive electric field along the domain (the $x$-direction). b) Distribution function of the resistive electric field.

associated with the energetic particles. In Sect. 4 we discuss possible consequences of removing these limitations.

Since the magnetic and the electric fields are given only at a discreet set of points (the grid-points of the MHD simulation), fields are interpolated with local 3-D linear interpolation. This provides the field values in between grid-points wherever they are needed for the integration scheme in order to achieve a prescribed precision.

\section{Results}

The model described in Sect. 2 is used to examine the acceleration of particles. In each example discussed, 30000 particles are injected with an initial Maxwellian distribution with a temperature of $1.2 \times 10^{6} \mathrm{~K}$ (the dashed curve in Fig. 3). The initial positions and pitch-angles of the particles are random.

We begin by describing a sample case that uses the parameters discussed at the end of Sect. 2. Figure 3 shows the final 


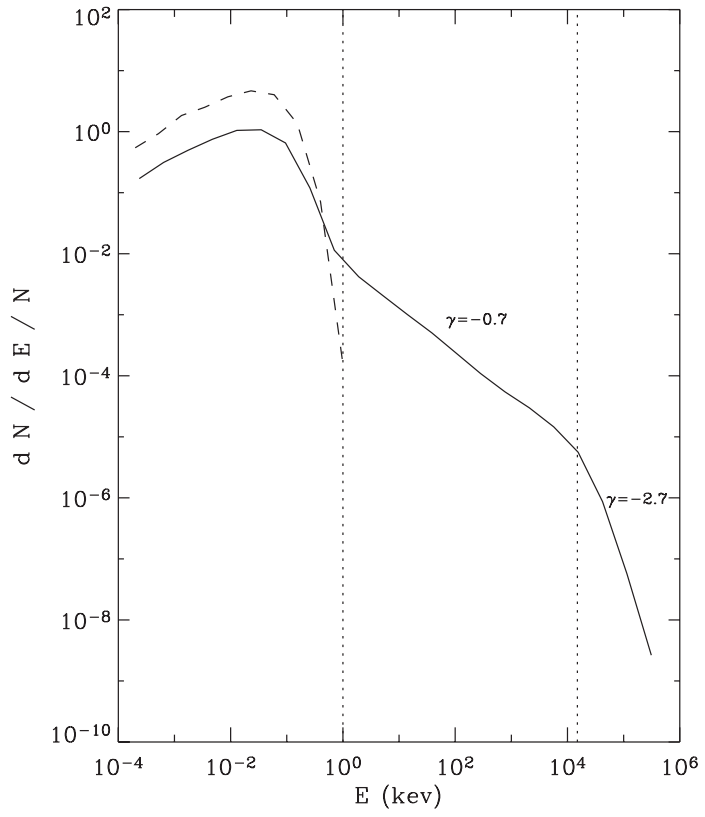

Fig. 3. Distribution function for all the particles injected in the domain at $t=0$ (dashed curve) and at the end of the run (solid curve). $\gamma$ is the power-law index value.

distribution function at $t=0.5 \mathrm{~s}$. The energy used to construct the distribution is either their final energy, or that with which they left the domain, as discussed in a moment. This distribution function has three main parts, the thermal part, and the two power law components with indexes equal to -0.7 and -2.7 respectively. Two or more of these components are observed in the majority of flares spectra (e.g. Lin et al. 2003). This distribution is in fact comprised of a number of "classes" of particles which behave differently throughout the simulation. In particular, particles can leave the domain through either the sides, or the ends, or become confined to the corona with or without energisation. These are discussed more fully in Sect. 3.2, but we first explore the dynamics of individual particles.

\subsection{Particles dynamics}

To investigate the physics behind the distribution function we first look at the dynamics of individual particles. While the magnetic field is mainly responsible for guiding the trajectory of the particles, it is the electric field at the current sheets that determines the energy gain or loss. Since the domain consists of regions of electric field pointing towards either of the footpoints, and regions with no electric field at all, from the viewpoint of a single charged particle it can be viewed as a domain of accelerating, decelerating and energy preserving regions.

Two different types of behaviour are evident.

If a particle travels over the different domains, gaining or loosing energy through a stochastic process through which any energy loss phase dose not exhaust all the energy that has being previously given to the particle, then the particle ends up leaving the box with a net energy gain (Vlahos et al. 2004 have shown how this stochastic process can be understood and modelled as a random walk in position and velocity space).
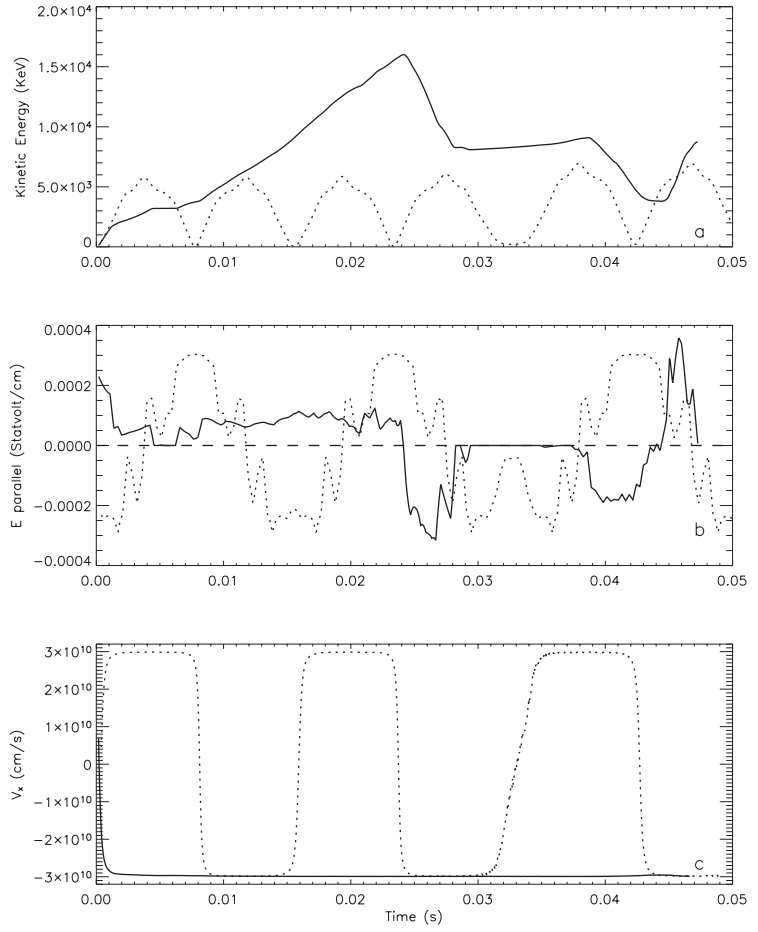

Fig. 4. a) The Energy gain versus time for a randomly chosen sample of 2 electrons; a trapped electron is shown with dotted lines and non-trapped one with solid lines. b) The parallel electric field experienced by the same two electrons. c) The velocity component along the domain $\left(v_{x}\right)$.

The solid curves of Fig. 4 shows the energy, instantaneous electric field and velocity of such an electron. Although the electron sees both positive and negative electric fields, the net result is a gain of energy. However, if any energy loss phase exhausts the entire energy of the particle, it gets trapped; it goes through a reversible process of loosing and gaining energy at the deceleration and acceleration sites. This second scenario is shown in the dotted curves in Fig. 4 where the particle is seen to gain and then lose all its energy. In an evolving background magnetic field, we would expect some of these particles to get de-trapped due to field line motions. However, as noted in Sect. 2 the acceleration timescale is very much faster than the MHD timescale, so trapping is in fact likely to occur on the acceleration timescale.

Figure 5, shows the trajectories of 50 randomly selected electrons. Trajectories that reach the sides and ends of the domain are the accelerated non-trapped particles, whereas trajectories that remain within the box are either the non-accelerated particles (these appear as very short trajectories) or the trapped particles.

\subsection{Electrons versus lons}

The overall dynamics of accelerated protons and electrons as they move through the electric fields are similar and their distribution functions look similar provided the respective particles are tracked for long enough within models that have the same parameters. 


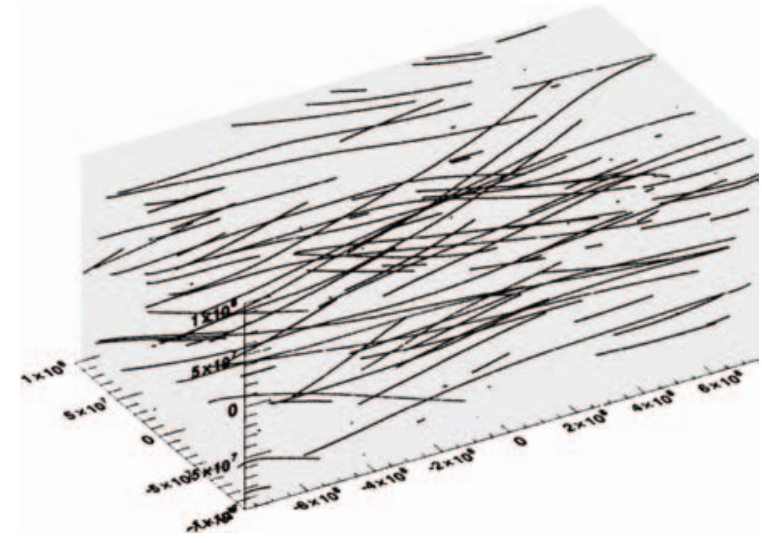

Fig. 5. The trajectories of 50 randomly selected electrons. It is evident that some particles leave both from the sides and the ends, while others are confined to a relatively small part of the domain.

However, the time needed to accelerate the two species to the same energies is not the same. For electric field acceleration by a highly super-Dreicer field, one might expect the ratio of the acceleration times for the two species to be in the order of the mass ratio. In fact this is only true in the highly sub-relativistic regime. Electrons in our model easily reach the relativistic regime, but protons take longer. As a result, the ratio of acceleration time between protons and electrons is not constant. Acceleration time is mainly dependant on velocity, which in highly sub-relativistic regime increases linearly with energy. Beyond that it increases in a slower base than energy due to relativistic effects. This is explained more in details in Sect. 3.4. For instance, for the parameters used here, electrons get accelerated to the maximum energy of $6 \times 10^{5} \mathrm{keV}, \sim 6$ times faster than protons. Figures 2 and 3 of Turkmani et al. (2005) showed that 10 randomly selected electrons and protons reached almost the same maximum energy level, but protons needed more time than electrons to do so.

Another difference between the two species is found when the inductive electric field is included. Through its perpendicular component this field leads to particle drift that is independent of charge and mass. However, the ratio of this drift velocity to the initial velocity of the particle is much larger for protons than electrons since for the same given temperature electrons travel faster than protons. This leads to a much more significant drift for protons than electrons leading to the protons initially moving in a direction perpendicular to the field lines, then gradually bending to move along the field lines when the parallel gain in energy exceeds the perpendicular one. This change in the directionality of the velocity was also seen in Dmitruk et al. (2004), however we found that the increased perpendicular velocity does not lead to a significant change in the net energy gain since the parallel acceleration remains more powerful than the perpendicular one, especially since in our case relativistic effects are included leading to the particles reaching higher energies unlike Dmitruk et al. (2004).
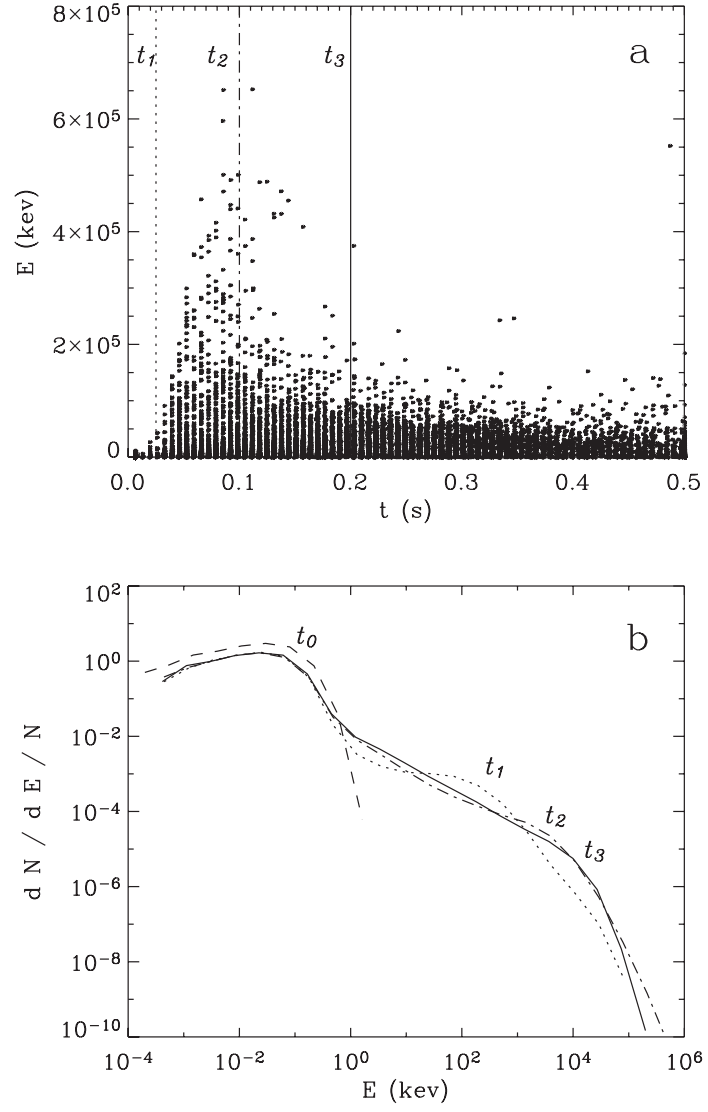

Fig. 6. a) The energy of protons as they leave the computational domain as a function of time. Each dot represents a single particle. b) The distribution functions for each of the times shown in Fig. 2a. The time $t_{0}$ corresponds to the start of the run.

\subsection{Spatial distribution and particle loss}

We have established that there are different "classes" of particles being accelerated, and so one expects the total distribution to be a convolution of them. Particle loss from the simulation domain is an important aspect of the model, and occurs continually. Figure 6a shows the energy of particles (protons in this example) as they are leaving the domain. In terms of the maximum energy of the escaping particles, two main phases can be distinguished: a rising phase from the beginning of the run to $t=t_{2}$ where a peak in the energy is reached followed by a decaying phase that lasts until the termination of the run.

The time at which a particle leaves the domain increases with; (a) the distance between the initial injection site and the escaping site at the borders of the domain, and (b) with the net acceleration a particle receives and how early it receives it. The net acceleration itself is governed by the net accelerating electric field a particle encounters, which in turn is dependant on the probability of the particle encountering acceleration or deceleration regions and the value of the electric field in these regions.

To help understand these scenarios and the differences between the different phases, we show in Fig. 7 the energy evolution of four typical particles from the different phases.

In the initial rising phase acceleration is dominant. This is because, even if a particle is injected to move in a direction that 


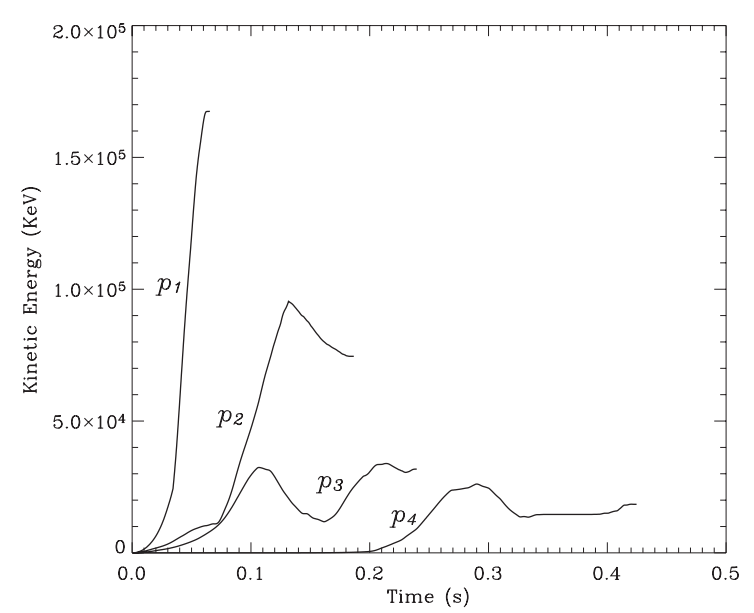

Fig. 7. Energy versus time for four selected protons representing the typical behaviour of particles from different phases in Fig. 6 .

leads to an energy loss, the particle, after decelerating and losing its initial thermal energy, bounces back to the energy gain direction, in which case and for the majority of the particles the energy gain far exceeds the low initial one. Many particles escape the domain straight after that. Such particles form the rising phase in which energy increases with time since particles need more time to get accelerated to higher energies. Particle $p_{1}$ in Fig. 7 is an example of this case.

If the particle remains inside the domain it then encounters other sites and become subject to deceleration as well as acceleration, most of the particles in the decaying phase follow this scenario. In this phase deceleration becomes an important factor; particles lose a portion of their energy and get delayed in escaping from the domain. The more they lose, the later they leave and thus the decrease in energy with the increasing time in this phase. Particles $p_{2}, p_{3}$ and $p_{4}$ in Fig. 7 are examples of this case. The zero electric field regions also act in delaying the particles in escaping the domain. Distribution functions for each of the four times shown in Fig. 6a are shown in Fig. 6b, where the different vertical lines in the top panel correspond to the distribution functions in the lower one. $t_{1}$ is chosen to be within the rising phase, $t_{2}$ is the time at which the energy gain reached its maxima and $t_{3}$ is chosen to be within the decaying phase.

The particles included in each distribution are the ones that remain inside the domain at that instant including the trapped ones. The dashed curve shows the initial Maxwellian distribution and as time increases, a portion of these particles are accelerated. In the rising phase up to $t_{1}$, the escaping particle distribution does not form a clear power-law, but by $t=t_{2}$, a pronounced double-power-law component forms whose first part terminates at $10^{4} \mathrm{keV}$, while the second steeper part extends beyond there. In the decaying phase at $t=t_{3}$ the power law components become smoother, but the indices have changed. The distribution also terminates at lower energy.

At the end of the run almost $40 \%$ of particles has left the domain through the footpoints, $13 \%$ through the sides, while the rest remain inside: $26 \%$ of those remaining have approximately thermal energies. Due to the inevitable predominance of one sign of electric field in the corona, an imbalance in the

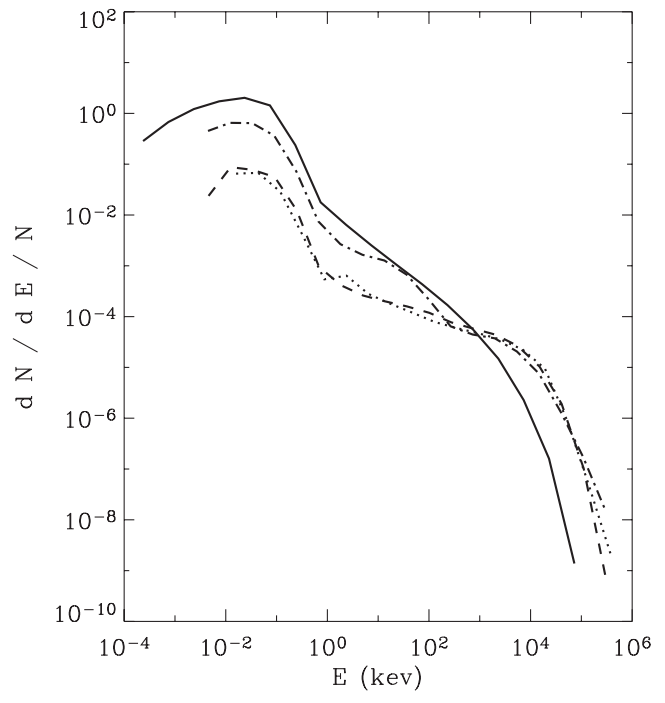

Fig. 8. Distribution functions in dotted, dashed and dashed-dotted curves for particles that leave from the right footpoint, left footpoint and the sides of the domain respectively, while the solid curve is for particles remaining inside the domain.

number of ions and protons at each footpoint is also present. For example, in the model being discussed here, $15 \%$ more electrons leave one footpoint than the other (which in turn attracts $15 \%$ more proton). This imbalance could be more or less extreme depending on the current sheets developments inside the domain, and so different snapshots of the MHD model will give different results.

In Fig. 8, distribution functions for these different categories of particles are shown. The dotted, dashed and dasheddotted curves are those of particles that leave from the right footpoint, left footpoint and the sides of the domain respectively, while the solid curve is for particles remaining inside the domain. It is obvious from the solid curve that the vast majority of the thermal particles are those which remained inside the domain. The higher energy part of the curves, however, represents the trapped particles. The distribution functions of the escaping particles show that they do reach higher energies than those trapped and that in the case of particle leaving from the footpoints the power-law component of the distribution function are flatter than those of particles remaining inside or leaving from the sides.

Figure 9 addresses the question whether there is a preferred site for acceleration in the domain during the run is investigated. Taking a snapshot at $2 \times 10^{3}$ gyration times, we show the energy change per second $\left(\frac{\partial E}{\partial t}\right)$ as a function of position for a large number of randomly selected particles. There is no strong spatial dependency in either the energy gain or loss. It is obvious from this figure that there is more energy gain than loss in the system, resulting in a net acceleration.

\subsection{Scaling of results with size of coronal domain}

Changing the coronal length from that used above leads to different acceleration properties since the current sheet length also changes. To examine this dependency we vary the 


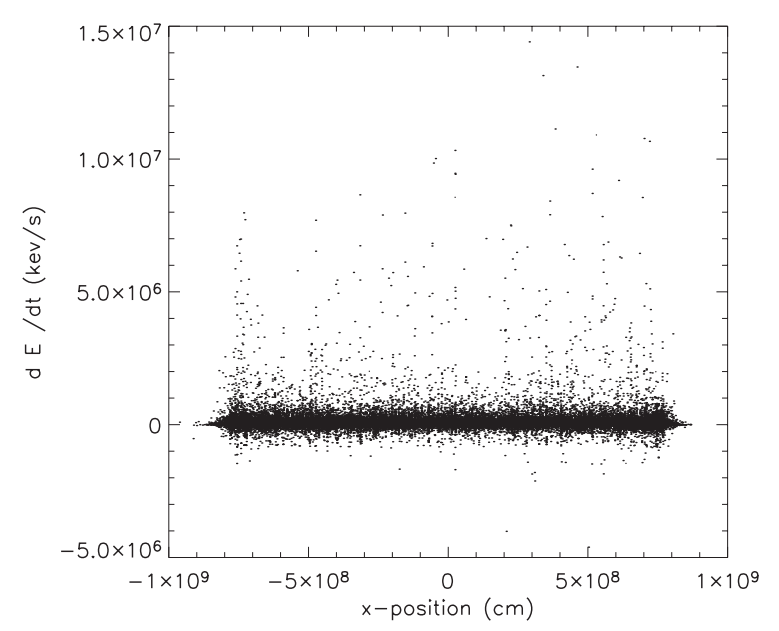

Fig. 9. $\frac{\partial E}{\partial t}$ for high number of randomly selected particles against the $x$-position along the loop.

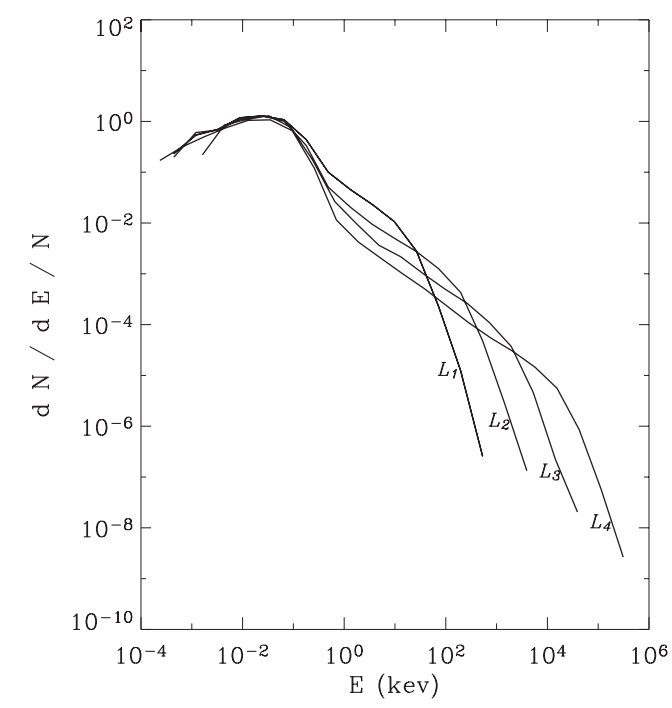

Fig. 10. Distribution functions for domains scaled with the length scales: $L_{1}=1.6 \times 10^{6}, L_{2}=1.6 \times 10^{7}, L_{3}=1.6 \times 10^{8}, L_{4}=1.6 \times 10^{9}$.

coronal length by the same proportion in all three dimensions. Six values of $L$ between $1.6 \times 10^{6}$ and $8 \times 10^{9} \mathrm{~cm}$ are considered. While some of the lengths used are small by coronal standards, their inclusion is important in establishing scalings of the acceleration process with length. The distribution functions are shown in Fig. 10 and the peak energy $\left(E_{\mathrm{p}}\right)$ and the time taken to reach that energy $\left(t_{\mathrm{p}}\right)$ are shown in Figs. 11a,b (c,d) for protons (electrons).

While the distribution functions in general retain their main features of possessing a thermal part and a double-power-law, the position of the high energy cut-off and thus the energy reached scales up linearly with the length scale. This property is shown in more details in Fig. 11. It is clear from Fig. 11b and d that there exist a linear relationship between the energy reached and the length for both electrons and protons. However, the scaling of $t_{\mathrm{p}}$ with length is not the same; below relativistic energies the relationship takes the form of constant acceleration $\left(t \propto L^{1 / 2}\right)$ and gradually changes to one with constant velocity at relativistic energies $(t \propto L)$. This is clear in the case of electrons in Fig. 11c since electrons become relativistic at $511 \mathrm{keV}$, while protons have to reach much higher energy. The above scaling can be explained by the relativistic equations of motion where the acceleration is governed by:

$\frac{\mathrm{d} \boldsymbol{v}}{\mathrm{d} t}=\frac{e}{\gamma m} \mathcal{E}+\frac{e}{\gamma m c} \boldsymbol{v} \times \boldsymbol{B}-\frac{e}{\gamma m c^{2}} \boldsymbol{v}(\boldsymbol{v} \cdot \mathcal{E})$,

with $\gamma=1 / \sqrt{1-v^{2} / c^{2}}$, with $\mathcal{E}$ the electric field, $c$ the speed of light, $m$ and $e$ the mass and the charge of the test-particle respectively and $E$ the electric field. The kinetic energy gain on the other hand is governed by

$E=(\gamma-1) m c^{2}$.

With $v$ increasing from 0 to $c, \gamma$ changes from 1 to infinity, therefore the increase in velocity is almost terminated once the particle is relativistic while the increase in energy keeps increasing with $\gamma$.

Comparing Figs. 11a and $\mathrm{c}$ we see that the ratio of the acceleration time for protons and electrons decreases with length, starting from $\frac{t_{\mathrm{p}-\mathrm{p}}}{t_{\mathrm{p}-\mathrm{e}}}=17$ for the shortest loop, ending with $\frac{t_{\mathrm{p}-\mathrm{p}}}{t_{\mathrm{p}-\mathrm{e}}}=6$ for a loop with length $1.6 \times 10^{9} \mathrm{~cm}$. This is also explained by a relativistic effect.

\subsection{Discussion of acceleration processes}

The main questions that arise from the results shown in Figs. 3-8 are; why is a thermal part of the distribution preserved, why is there a power-law dependency and what determines the location of the high-energy cut-offs and the maximum energy reached? As we will show the total distribution function is the sum of particles that interact with the electric fields in different ways.

The preservation of the thermal component is accounted for by the fact that not all the particles are injected at or near a current sheet, or may not interact with one. For such particles the energy remains constant. Also, as noted above, some particles are decelerated towards thermal velocities after their initial acceleration. (We note here that when the inductive electric field was included in Turkmani et al. 2005, it lead to a low energy cut-off that the resistive electric field alone could not account for. Despite the inductive field being in general negligible, in the low energy regime its importance stems from a spatial distribution that is less confined than the resistive field. Particles that do not encounter these current sheets can still get limited acceleration from the inductive field. This effect may well explain the low energy cut-off that is observed in some flares (e.g. Holman \& Benka 2002; Holman et al. 2003).)

Since the net acceleration is dependant on the value of the net electric field, the acceleration is expected to scale similarly to the electric field. The latter as shown in Fig. 2 posses a power-law component which could explain the power-law component in the energy distribution function.

The energy at which the spectra terminate is determined by the overall size of the computational domain. Scalings with the parameter $L$ are discussed in the previous section. As explained earlier, the accelerated particles either become trapped or are free to roam the domain. The latter particles have the 

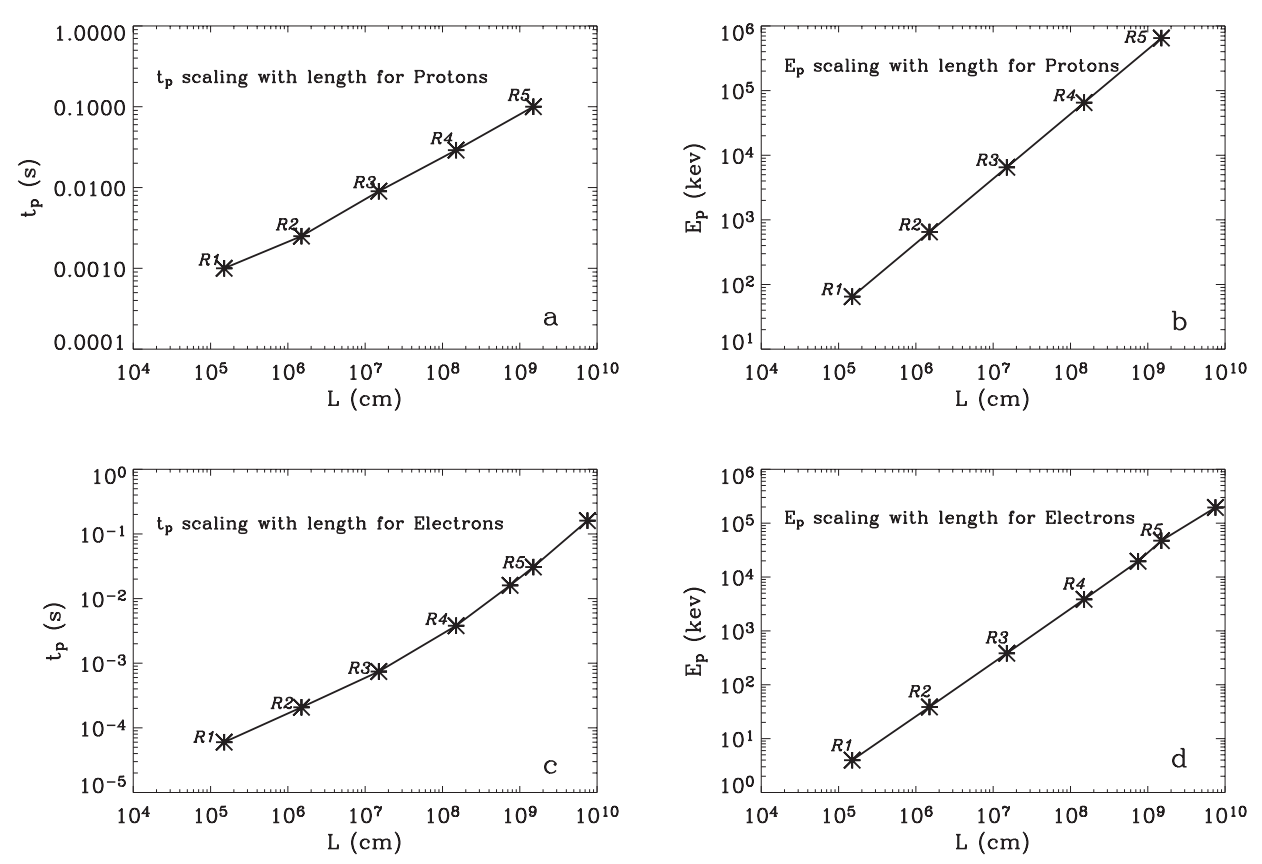

Fig. 11. $t_{\mathrm{p}}$ versus $L$ for protons a) and electrons c). $E_{\mathrm{p}}$ versus $L$ for protons b) and electrons d).

opportunity to encounter more acceleration regions and thus reach higher energies. The trapped ones on the other hand remain confined to one region which limits the maximum energy they can reach. The non-thermal part of the solid curve in Fig. 8 corresponds to the trapped accelerated particles. This component terminates near the high-energy cut-off of the total distribution function, meaning that what lies beyond this point in the total distribution consist of almost entirely escaping particles. This partially explains the break in the power-law in Fig. 3. The second reason for this break is an existing one in the distribution function of the resistive electric field itself (as shown in Fig. 2) which leads to breaks in the distribution functions of the trapped and escaping particles.

Looking at the statistics of the trapped and non-trapped particles it is found that the first power-law part represents the trapped particles in addition to half of the non-trapped accelerated ones: this part terminates at a maximum energy determined by the scale of the biggest current sheet. Beyond this point the second half of the distribution function represents the other half of the non-trapped accelerated particles that reached beyond this energy.

As it has been shown in Sect. 3.4, the location of the points discussed above is linearly dependant on the size of the system, a longer domain means longer current sheets as well (at least in our case since we are rescaling from one MHD model) and thus the change of the size of the system does not only change the maximum energy it reaches but also the location of the highenergy cut-off (which also scales linearly with the length scale).

\subsection{The $X$-ray spectra}

We have evaluated the thick target bremstrahlung X-ray spectrum from the distribution function of the accelerated electrons using the Haug (1997) bremsstrahlung cross section to
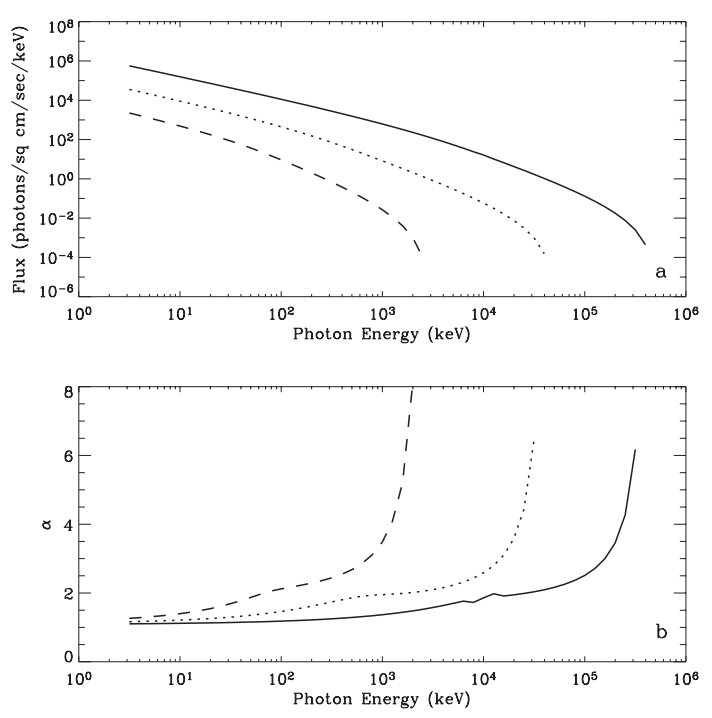

Fig. 12. a) The thick target bremstrahlung signature for the accelerated particles for loops with lengths $L_{1}=1.6 \times 10^{7}$ (dashed curve), $L_{1}=$ $1.6 \times 10^{8}$ (dotted curve) and $L_{4}=1.6 \times 10^{9}$ (solid curve) $\mathrm{cm}$. b) The absolute value of the index $\alpha$ for the spectra in a).

calculate the photon flux using Brown (1971) formulae. (Note that our model cannot address properly the evolution of the distribution function as it leaves the corona.) The resulting spectra from the models with three different length scales are shown in Fig. 12. While the two parts power-law feature remains conserved, the index of the slopes changes.

At low energies (below $1 \mathrm{MeV}$ ) the indices take an absolute value between one and two, whereas at higher energies, they are larger, between 2 and 5 . (Note the $\sim-1$ change in the index for the first power-law component value compared to that of the distribution function, as has been noted before by Brown (1971): Eqs. (14) and (15).) However, the low energy indices 
are significantly smaller than those observed (e.g. Aschwanden 2002), indicating that the acceleration mechanism discussed here is more efficient as the energy increases than in reality. We discuss possible resolutions of this problem in Sect. 4.

However the distribution functions and spectra show some generic similarities to the observed one, which possess in most cases thermal and power-law components, and in many cases a double power-law structure (e.g. Holman et al. 2003). The extension of the spectrum and the position of the high-energy cut-off though may vary depending on factors like the length scale as explained in Sect. 3.4. These features of our spectra stem directly from those of the distribution functions since no propagation physics is taken into account here, and therefore explaining the features of the distribution functions accounts for those in the spectra.

\section{Discussion and conclusions}

In this paper, we present a study of particle acceleration in a stressed coronal magnetic field. Following on from our earlier brief letter Turkmani et al. (2005), we use electromagnetic fields calculated from a three-dimensional MHD code as input for a test particle simulation of electrons and protons. Particles gain or lose energy in a stochastic process due to their interaction with different current sheets. While most of the particles finally leaving the computational domain with a (often significant) net energy gain, some remain trapped in the coronal fields, and may have no energy gain. Electrons are accelerated faster than protons.

The stressed coronal fields are a very effective particle accelerator, with both electrons and protons attaining relativistic energies in a very short time throughout the corona: for example electrons are accelerated to relativistic energies in milliseconds. The acceleration appears to have four phases, with the maximum energies rising, peaking and then decaying, as well as there being an extended acceleration phase lasting for almost $1 \mathrm{~s}$. The energy reached scales with the coronal length scale.

The observed spectral index of hard X-ray emission is shown to be time dependant (e.g. Grigis \& Benz 2004). We find that the index is dependant on energy and increases towards higher energies. However the temporal dependency is difficult to examine on the MHD time scale and is only limited to be examined on the kinetic time-scale. On that scale we see behaviour similar to the observed raise-peak-decay phases followed by an extended acceleration phase. The spectral indexes we found to be changing with these phases, but a difficulty is that the observed thick target spectrum is steeper than our calculated one.

While this paper has demonstrated the effectiveness of particle acceleration in stressed coronal fields, it has raised significant questions that must serve to guide future research on this topic. Hints of the limitations come from the shallow particle and photon spectra which indicate that the acceleration may be too efficient. The key question is that of feedback from the accelerated particles to the MHD equations. Although we are in the test particle limit, it is clear from the fact that many particles are accelerated that the energy in the energetic particles can become significant compared to that in the coronal electromagnetic fields. Conservation of energy requires that as the particle energy increases, the energy in the electromagnetic fields must decrease. This in turn must imply weaker electric fields, and so less efficient acceleration. One would anticipate in turn that this would lead to fewer particles at higher energy and so steeper spectra. Note that since we expect the acceleration to be fast, any photospheric driver cannot replenish the coronal magnetic energy. However, these conjectures require a new generation of models which are under development at this time.

Finally, we note that an MHD snapshot has been used. Future work will need to examine the acceleration of particles in a series of MHD snapshots to examine the time variability of the energetic particles. We recall that the variability in the MHD fields need not occur on the photospheric timescales, but can be much faster if the stressed coronal state relaxes rapidly to try to find a new equilibrium. However this should still be longer than the acceleration timescale.

Acknowledgements. This work was funded by the European Commission under research training network HPRN-CT-2001-00310. K.G. acknowledges funding by PPARC in the form of an Advanced Fellowship and Carlsbergfondet in the form of a Scholarship over various periods of the project. P.C. acknowledges the support of a PPARC senior fellowship. The software used in the paper to calculate the Bremstrahlung spectra is from the solar software library.

\section{References}

Anastasiadis, A., \& Vlahos, L. 1994, ApJ, 428, 819

Anastasiadis, A., Vlahos, L., \& Georgoulis, M. K. 1997, ApJ, 428, 819

Anastasiadis, A., Gontikakis, C., Vilmer, N., \& Vlahos, L. 2004, A\&A, 422, 323

Arzner, K., \& Vlahos, L. 2004, ApJ, 605, L69

Aschwanden, M. 2002, Space Sci. Rev., 101, 1

Brown, J. C. 1971, SoPh, 18, 489

Dmitruk, P., Matthaeus, W. H., Seenu, N., \& Brown, M. R. 2003, ApJ, 597, L81

Dmitruk, P., Matthaeus, W. H., \& Seenu, N. 2004, ApJ, 617, 667

Galsgaard, K. 2002, SOLMAG 2002, Proc. the Magnetic Coupling of the Solar Atmosphere Euroconference and IAU Coll., 188, ESA SP-505

Galsgaard, K., \& Nordlund, A. 1996, J.G.R., 101, 13445

Grigis, P. C., \& Benz, A. O. 2004, A\&A, 426, 1093

Haug, E. 1997, A\&A, 326, 417

Hendrix, D. L., \& Van Hoven, G. 1996, ApJ, 467, 887

Holman, G. D., \& Benka, S. G. 1992, ApJ, 391, 854

Holman, G. D., Sui, L., Schwartz, R. A., \& Emslie, G. 2003, ApJ, 595, 97

Hurford, G. J., Schwartz, R. A., Krucker, S., et al. 2003, ApJ, 595, 77

Lin, R. P., Krucker, S., Hurford, G. J., et al. 2003, ApJ, 595, L69.

Miller, J. A., Cargill, P. J., Emslie, A., et al. 1997, JGR, 102, 14631

Nordlund, A., \& Galsgaard, K. 1997, A 3D MHD Code for Parallel Computers, Technical Report, Astronomical Observatory, Copenhagen University

Turkmani, R., Vlahos, L., Galsgaard, K., \& Isliker, H. 2005, ApJ, 620, L59

Vlahos, L., Isliker, H., \& Lepreti, F. 2004, ApJ, 608, 540 